\title{
Breaking the rules: understanding non-compliance with policies and guidelines
}

Healthcare organisations use policies and guidelines to standardise and clarify care and improve efficiency, productivity, and safety. But Jane Carthey and colleagues are concerned that their burgeoning number makes it impossible to distinguish the essential from the irrelevant and is affecting compliance

\author{
Jane Carthey human factors consultant ${ }^{1}$, Susannah Walker anaesthetic registrar ${ }^{2}$, Vashist Deelchand \\ research associate ${ }^{2}$, Charles Vincent professor of clinical safety research ${ }^{2}$, William Harrop Griffiths \\ consultant anaesthetist ${ }^{3}$
}

${ }^{1}$ Imperial College London, London, UK; ${ }^{2}$ Department of Biosurgery and Technology, Imperial College London; ${ }^{3}$ Department of Anaesthesia, Imperial College Healthcare NHS Trust, London

Healthcare staff in the National Health Service are expected to comply with and keep up to date with numerous policies covering every aspect of their daily work. The National Institute for Health and Clinical Excellence (NICE) has guidelines on a huge number of clinical issues ranging from how to treat breast cancer to how to insert a central venous catheter. ${ }^{12}$ Other guidelines are generated by external bodies such as the royal colleges and professional bodies, the General Medical Council, or the Care Quality Commission and reflect the external regulatory framework in which healthcare operates. The NHS Litigation Authority requires trusts to have clinical governance and risk assessment policies in order to get a discount on their insurance contributions. Additionally, many guidelines are locally generated and cover routine activities and the management of different types of risk. Although policies and guidelines are important, the large number of guidelines and many different sources make it impossible for staff to comply with all of them.

\section{Information overload}

As part of a research project aimed at understanding the causes of non-compliance, we identified guideline publishers and also counted the number of policies and guidelines on three trust intranet sites. The former NHS Library had a list of 152 publishers of guidelines and 17 references to guidelines about how to develop guidelines.

The number of guidelines and policies on the intranets of three central London NHS acute trusts varied between 192 and 457. In addition to these trust specific guidelines, staff have to understand and comply with local, professional, and governmental policies and guidelines.
To illustrate how this glut of policies and guidelines affects clinical staff in practice we provide two examples. The first outlines a typical journey of an elderly patient admitted for emergency surgery on a fractured neck of femur (figure $\Downarrow$ ). An estimated 75 clinical guidelines and trustwide policies cover the different stages of management. The second example describes the challenge facing a clinical director of anaesthetics who wants to ensure that clinical staff are aware of and compliant with all relevant guidelines. We identified 21 organisations that publish guidelines related to anaesthesia (box 1).

A clinical director of anaesthesia would start by reviewing guidelines published by the Royal College of Anaesthetists and the Association of Anaesthetists of Great Britain and Ireland, which both produce effective and relevant guidelines. Together, their guidelines encompass 80 different topics, most of which relate to personal clinical responsibility, although some cover organisational managerial issues. The clinical director, together with departmental colleagues, will then decide which guidelines are relevant to the department and how these will be disseminated.

The clinical director will also have to consider the guidance from government bodies such as the Department of Health and NICE. Unfortunately, both have complex websites with vast numbers of guidelines (over 3000 from the health department and more than 1000 from NICE). Neither has an anaesthesia or critical care section. Therefore a time consuming trawl through all possibly relevant guidance is required. The NICE website contains guidance relevant to anaesthesia in many different sections-for example, guidance on the prevention of surgical site infection is in the infectious diseases section and guidance 


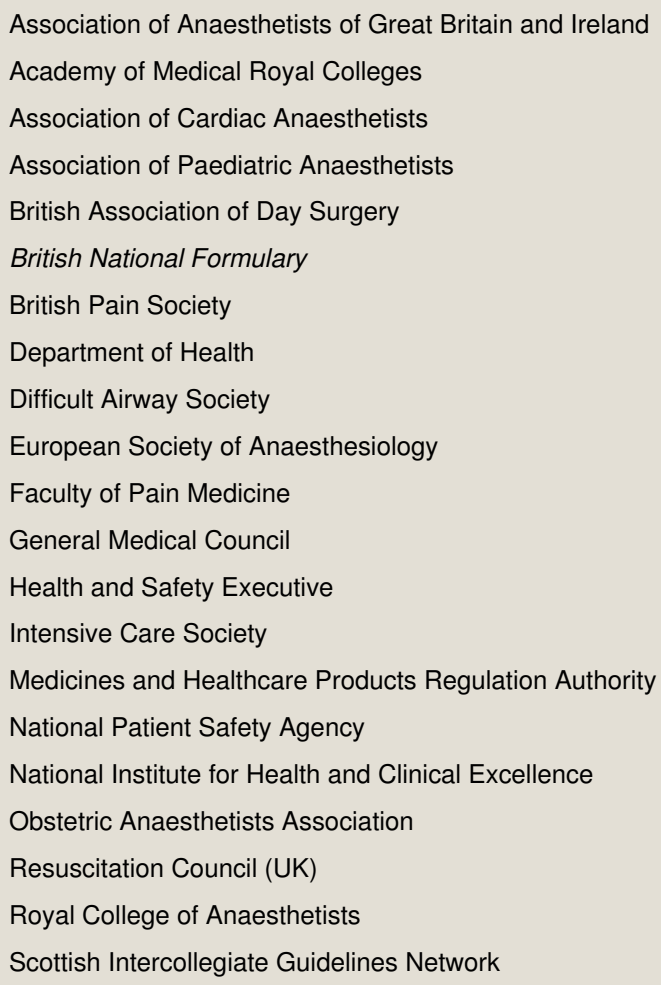

on management of diabetes in pregnancy is in the endocrine section.

The Department of Health website is even more challenging to navigate, with guidance listed under varying titles of Good Practice, Protocols, Procedures, Standards, Guidelines, and Publications. When the research team reviewed the website, guidance about the correct method of decontamination of surgical equipment was displayed on the same webpage as guidance about how social services departments should communicate with the general public.

The next step would be to consult bodies relevant to specialist types of surgery performed in the department, such as cardiac, paediatric, and obstetric anaesthesia.

Finally, there are additional areas in which guidance should be sought. For example, all trainees should be aware of the UK Resuscitation Council's guidance. They should also be aware of guidelines published by the Intensive Care Society as these cover various areas managed by anaesthetists, such as the management of patients after cardiac arrest, transfer of critically ill patients, and issues surrounding organ donation.

In summary, the complexity of the current system makes it difficult for a clinical director of anaesthesia to find all relevant policies and guidelines, still less to disseminate and implement them. Full compliance is in practice impossible.

\section{Unintended consequences of too many rules}

\section{Volume}

Navigating through the myriad policies and guidelines is complicated and time-consuming. Similarly, disseminating the information in a way that ensures the relevant people are aware of the latest policies and guidelines is equally challenging. The constant barrage of guidelines lessens their impact and reduces compliance with the more important ones.

\section{Multiple rules on the same topic}

Multiple policies and guidelines often exist on the same topic. For example, the American Association of Clinical Endocrinologists and the International Diabetes Foundation have both published comprehensive guidelines for management of diabetes. ${ }^{3}$ Healthcare professionals are then unsure about whose guidelines to follow.

\section{Naming and accessibility}

Policies tend to be located on hospital intranets, although some departments have chosen to store them on specific "drives" on the departmental computers. However, this assumes that staff have easy access to computers, which is often not the case, and requires them to know the name of the policy they are searching for and where to find it. The operating theatres we assessed store their policies on a separate "J drive" within the local IT system. However, very few members of staff questioned were aware of this fact. Confusion is also created by the obscure wording of some policy titles. What do you think the "Acceptable Use Policy" refers to? Its aim is to explain what constitutes acceptable use of a trust's internet.

\section{Length and complexity of guidance}

Organisations often try to specify every conceivable aspect of care in order to protect themselves from litigation or other sanctions. This results in long, wordy policies that are hard to navigate. We identified a 122 page "medicines policy" and 120 page "safe handling of healthcare waste policy." The operating theatre staff we interviewed during our research commented that they could "never find the controlled drugs section" within the medicines policy.

National guidance can also be long and complex, with each successive version being longer than the last. For example, the most recent government guidance on child protection, Working Together to Safeguard Children, is 390 pages long, ${ }^{4}$ leading 
one author to comment on the exponential growth in the complexity of national safeguarding guidance over the past 30 years. ${ }^{5}$

\section{Trivial policies}

New policies are sometimes created as a "knee jerk" response to a specific incident. This can lead to the development of a range of trivial policies covering the wearing of Crocs in theatres, managing adverse weather conditions, how healthcare staff should answer a telephone, and how to politely "meet and greet" visitors to a hospital department. Such policies may affect staff morale and their willingness to comply with other important policies because the organisation's policies are perceived as "just another dictat from above."

\section{Version control}

Policies and guidelines often need to be updated, but the previous versions may remain in circulation. Different versions may then be stored in a variety of places, confusing staff and diluting their impact. Staff will be uncertain about which policy to follow or may be unaware of the new version's existence. For example, a report into an accidental death from spinal injection of vincristine found that there were two versions of the haematology guidelines issued for the ward. ${ }^{6}$ The protocol also stated that, "There is a more detailed protocol for the administration of cytotoxics," but did not give the name or location of this critical document.

\section{How can healthcare improve current practice?}

Length, complexity, accessibility, volume, and failure to consult with healthcare professionals who have to follow a policy all reduce compliance with potentially critical results. Staff may break the rules because of their complexity, may follow the wrong policy when there are multiple versions, or be completely unaware of a policy because of its obscure location or inadequate dissemination. Yet many employers will discipline staff for non-compliance if a patient is harmed. How can we produce usable policies and guidelines that support rather than burden staff?

Human factors research across several industries has shown that the more prescriptive rules workers have imposed on them, the less likely they are to comply. ${ }^{7}$ Humans are also naturally adaptable and tend to improvise, which makes some non-compliance inevitable. ${ }^{9} 10$

To improve compliance with policies and guidelines it is essential to understand why staff break the rules, to simplify and standardise guidance, and to design out factors that increase the risk of non-compliance. Healthcare needs to learn from the approach taken by other high technology industries. For example, in air traffic control the number of national bodies producing policies is rationalised, presentation is standardised, and air traffic controllers are engaged in writing standard operating procedures. There are also robust systems in place to monitor version control and ensure air traffic controllers read and understand standard operating procedures.

\section{Key actions at national and local level}

We suggest several actions that could be taken to reduce the complexity and proliferation of policies and guidelines in NHS organisations. Cooperation and collaboration between national bodies that set policy requirements is a prerequisite for reducing volume and conflicting requirements. Similarly, local healthcare organisations need to review existing policies and consider whether volume, version control, accessibility, length, or titling problems may increase the risk of non-compliance. Box 2 provides an example of good local practice from one London NHS foundation trust.

We suggest that human factors science be applied to the development, design, and testing of policies and guidelines; involving healthcare staff who have to follow the policy in the development phase will ensure they are usable in practice. Rather than sending the draft policy to a small group of experts to comment on, trusts should carry out walkthroughs and risk assessments aimed at identifying how the policy could be read and misinterpreted by those who have to use it.

Trusts should also learn from research on implementing quality improvement initiatives and evidence based medicine. The principles for getting clinicians to implement evidence based medicine also apply to improving levels of procedural compliance. If a clinician is aware of the evidence and the benefits, and if the implementation process is practical it is more likely to be adopted. ${ }^{11}$ Similarly, if healthcare professionals can see the need for a policy or guideline, if it is written in a way that shows a practical understanding of the real world, and if it is easy to access and follow, staff are more likely to comply with it.

Finally, both national and local organisations would benefit from adopting tracking mechanisms used by industries such as air traffic control which enable them to monitor whether staff have read and, more importantly, understood key messages.

Clinical policies and guidelines are undoubtedly an essential foundation of high quality patient care. However, their extraordinary and uncoordinated proliferation in the NHS confuses staff, causes inefficiencies and delay, and is becoming a threat to patient safety. We need to recognise the problems caused by current approaches and introduce greater rationalisation and standardisation at both national and local levels.

The Imperial Centre for Patient Safety and Service Quality is funded by the National Institute of Health Research. We thank staff from the orthopaedic department at Imperial College Hospitals NHS Trust and NHS Lothian for their help.

Contributors and sources: $\mathrm{JC}$ is a human factors expert who has worked in the NHS for 15 years. JC led the research project, wrote the initial and subsequent drafts of the paper, and led work in the London NHS foundation trust cited in box 2. CV is an international expert on patient safety. CV was the senior academic lead for the work and assisted with editing drafts of the manuscript. WHG is vice president of the Association of Anaesthetists of Great Britain and Ireland and has been a coauthor of a number of its practice guidelines. WHG commented on drafts of the paper and assisted with the development of the sections of the paper describing the clinical director of anaesthetics dilemma in understanding policies and guidelines. SW and VD collected the data on guideline publishers and developed the figure and box 1 . Information was collected from literature review, trust and national agency intranet sites, a previous project carried out at UCLH NHS Hospitals Foundation Trust and interviews with healthcare staff. JC is the guarantor.

Competing interests: All authors have completed the ICJME unified declaration form at www.icmje.org/coi_disclosure.pdf (available on request from the corresponding author) and declare the Imperial Centre for Patient Safety and Service Quality is funded by the National Institute of Health Research. CV has received funding from The Health Foundation and receives royalties from his book on patient safety.

Provenance and peer review: Not commissioned; externally peer reviewed. 


\section{Box 2: How one foundation trust is tackling non-compliance}

In a review of non-compliance carried out in 2009, the trust identified problems with the accessibility, length, volume, and complexity of trust-wide policies. The trust is currently implementing an action plan to improve levels of policy compliance by

- Applying "lean thinking" to simplify the local policy development and implementation processes

- Reducing the number of trust-wide policies

- Improving consultation with healthcare professionals when policies and guidelines are developed

- Piloting software that will improve the trust's ability to monitor that staff have read and understood key policy messages

- Raising awareness among senior managers to ensure that there is a better understanding that simply writing a policy does not reduce risk

- Revising key words and search teams on the trust's intranet site to improve accessibility.

Future work is planned to provide training in writing policy, developing e-learning on non-compliance so that staff better understand the rationale for each policy, and embedding discussions about policy non-compliance into the trust's programme of executive walk rounds.

1 National Institute for Health and Clinical Excellence. CG81: Advanced breast cancer: diagnosis and treatment. National Collaborating Centre for Cancer, 2009.

National Institute for Health and Clinical Excellence. Guidance on the use of ultrasound locating devices for placing central venous catheters. Technology appraisal guidance No 49. NICE, 2002.

3 National Institute for Health and Clinical Excellence. CG63: diabetes in pregnancy: management of diabetes and its complications from preconception to the postnatal period. National Collaborating Centre for Women's and Children's Health, 2008.

4 HM Government. Working together to safeguard children: a guide to the inter-agency working to safeguard and promote the welfare of children. Department of Children, Schools and Families, 2010.

5 Parton $\mathrm{N}$. The increasing length and complexity of central government guidance about child abuse in England, 1974-2010. http://eprints.hud.ac.uk/9906/.

6 Toft B. External inquiry into the adverse incident that occurred at Queens Medical Centre, Nottingham, 4th January 2001. Department of Health, 2001.

7 Reason JT, Parker D, Lawton R. Organisational control and the varieties of rule related behaviour. J Organisat Occup Psychol 1998;71:289-304.
8 Lawton R. Not working to rule: understanding procedural violations at work. Safety Sci 1998;28:77-95.

9 Amalberti R, Auroy Y, Berwick D, Barach P. Five system barriers to achieving ultrasafe health care. Ann Intern Med 2005;142:756-64.

10 Amalberti R, Vincent C, Auroy Y, de Saint Maurice G. Violations and migrations in healthcare: a framework for understanding and management. Qual Saf Health Care 2006;15:i66-71.

11 Glaziou P, Haynes B. The paths from research to improved health outcomes. ACP J Club 2005;142(2):A8-10.

Accepted: 4 August 2011

Cite this as: BMJ 2011;343:d5283

(c) BMJ Publishing Group Ltd 2011 


\section{Figure}

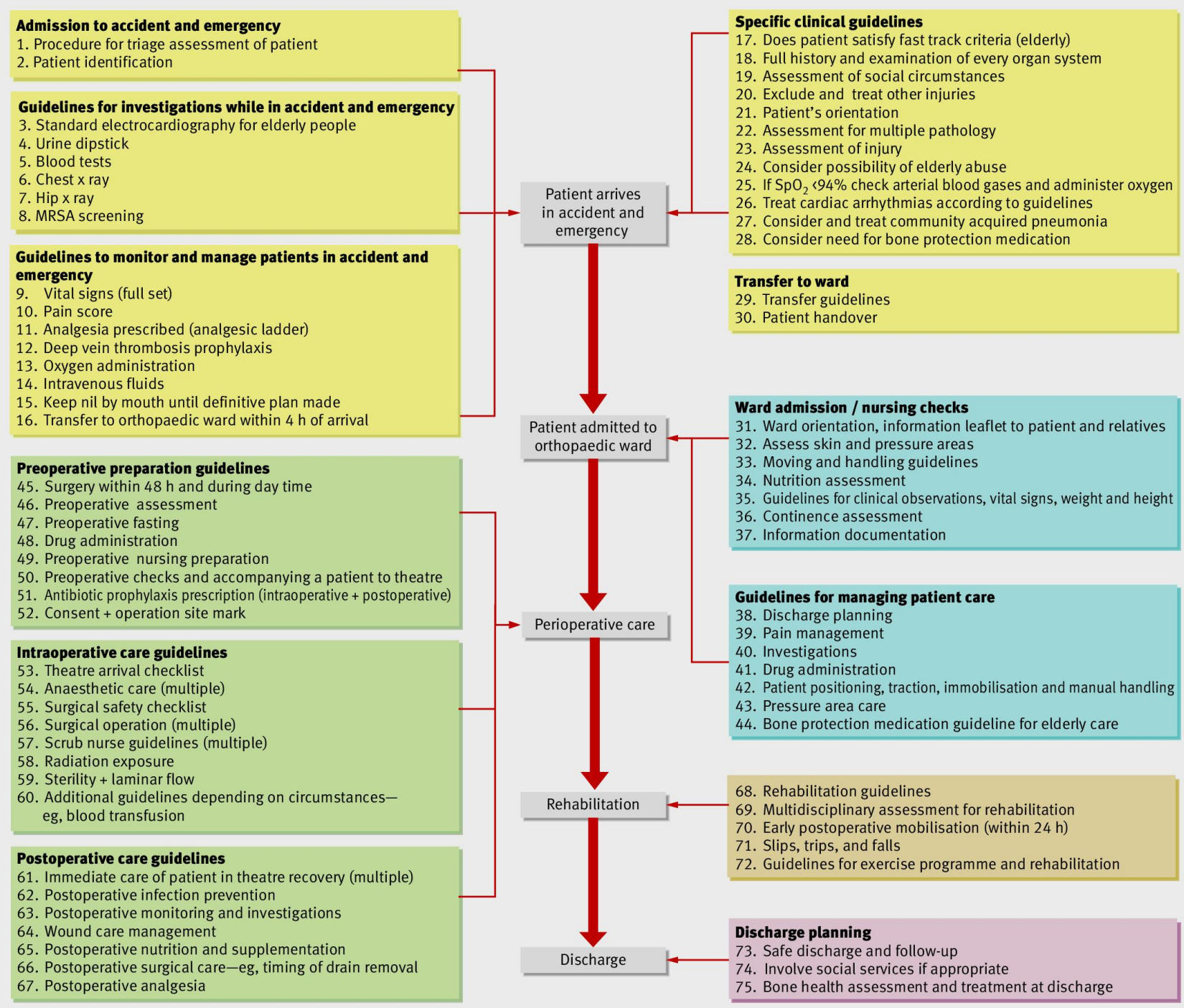

Fig 1 Typical patient journey for an elderly patient with fractured neck of femur 